\title{
DESIGN CALCULATION FOR CROSSFLOW GAS ABSORPTION TOWERS WITH HEAT GENERATION
}

\author{
YoSHISHIGE HAYASHI, EIJI HIRAI AND MASAKAZU OTAKI \\ Department of Chemistry and Chemical Engineering, Kanazawa University, Kanazawa 920
}

Key Words: Absorption, Crossflow, Tower Design, Heat Generation, Gas-Liquid Interface, Numerical

Calculation, Complex Method

\section{Introduction}

A number of industrially important gas absorption operations are strongly exothermic and the heat effect is considerable. In these cases, simultaneous heat and mass transfer must be taken into account.

Inazumi et $a{ }^{4)}$ have proposed a design calculation method for the nonisothermal crossflow gas absorption tower with solvent vaporization, and it has been pointed out that there are some difficulties in the estimation of concentration and temperature at the gas-liquid interface owing to the introduction of the difference method and the trial-and-error method. The design calculation for the nonisothermal gas absorption tower depends on whether the exact values at the gas-liquid interface can be estimated or not, and a very accurate numerical solution is required.

In this paper, a difference of the enthalpy of mixed gas presented by Inazumi et $a l^{4)}$ is used as a driving force and an exact design calculation method for the nonisothermal crossflow absorption tower is proposed. ${ }^{2,3}$ This method has the distinctive feature of applying a nonlinear optimization procedure. The concentration and temperature at the gas-liquid interface are calculated by the Newton-Raphson method, and the values can be estimated easily within an error of the order of $10^{-6}$ or less and the calculation time can be reduced. The design calculation is performed by the Complex method ${ }^{1)}$ to reduce the computation time, and when the allowable error for the criterion function in the Complex method is restricted strictly the calculated results are more reliable than those by the other method ${ }^{4)}$ because the calculation error is smaller and the precision is improved.

\section{Theoretical Consideration}

Figure 1 shows a schematic representation of a crossflow gas-liquid contactor with solvent vaporization. $X$ and $Y$ denote the axes in the direction of the

Received October 9, 1985. Correspondence concerning this article should be addressed to Y. Hayashi. M. Otaki is now with Otsuka Medical Co., Ltd., Tokyo 101. gas and liquid flows respectively, and $Z$-axis is perpendicular to the $X-Y$ plane.

To solve this problem, some assumptions have been made to reduce the complexity of the equations: (1) Gas and liquid flows are steady and plug flow, respectively. (2) The variations of $\bar{x}, \bar{y}, t$ and $T$ in the $Z$-direction are negligible. (3) The gas- and liquid-film volumetric coefficients, such as $k_{G} a, k_{L} a$, $k_{H} a, h_{G} a$ and $h_{L} a$ are constant throughout the tower. (4) The mass transfer rates for both gas and liquid phases are not affected by each other. (5) The absorption tower is adiabatic. (6) The mass velocity of solvent is constant in the tower.

\subsection{Basic equations}

Now, let us consider the mixed gas composed of three components, i.e. inert gas, solute gas and solvent vapor. In this case, the basic equations presented by Inazumi et al. ${ }^{4)}$ are used as follows:

$$
\begin{aligned}
& C_{H}=C_{a}+C_{b} \bar{y}+C_{v} H \\
& \bar{i}=C_{H}\left(t-t_{0}\right)+\left(Q_{0} / \beta\right) \bar{y}+(\alpha / \beta) r_{0} H \\
& -G C_{H} \partial t /\left.\partial X\right|_{Y}=h_{G} a\left(t-t_{i}\right) \\
& -L C_{P L} \partial T /\left.\partial Y\right|_{X}=h_{L} a\left(T-t_{i}\right) \\
& -G \partial \bar{y} /\left.\partial X\right|_{Y}=k_{G} a P\left(\bar{y}-\bar{y}_{i}\right) \\
& L \partial \bar{x} /\left.\partial Y\right|_{X}=k_{L} a \rho_{L M}\left(\bar{x}_{i}-\bar{x}\right) \\
& G \partial H /\left.\partial X\right|_{Y}=k_{H} a\left(H_{i}-H\right)
\end{aligned}
$$

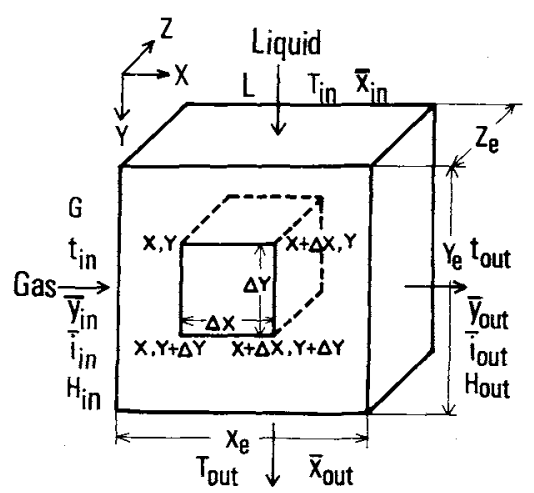

Fig. 1. Schematic model and coordinate system. 


$$
\begin{aligned}
& -G \partial \bar{y} /\left.\partial X\right|_{Y}=L \partial \bar{x} /\left.\partial Y\right|_{X} \\
& \left(\bar{i}-\bar{i}_{i}\right) /\left(T-t_{i}\right)=-h_{L} a C_{H} / h_{G} a \\
& \left(\bar{y}-\bar{y}_{i}\right) /\left(\bar{x}_{i}-\bar{x}\right)=k_{L} a \rho_{L M} / k_{G} a P
\end{aligned}
$$

where

$$
\begin{aligned}
& C_{a}=f_{1}(t), \quad C_{b}=f_{2}(t), \quad C_{v}=f_{3}(t) \\
& \bar{y}_{i}=f_{4}\left(\bar{x}_{i}, t_{i}\right) \\
& H_{i}=f_{5}\left(\bar{x}_{i}, t_{i}\right) \\
& \alpha=k_{H} a / k_{G} a P, \quad \beta=h_{G} a / k_{G} a P C_{H}
\end{aligned}
$$

\subsection{Numerical solution}

The dimensions of the tower are subdivided into several small parts as shown in Fig. 2 and the finite difference method is used. As a result, the following generalized forms are obtained.

$$
\begin{aligned}
& \bar{y}_{i_{I, J}}=m_{H}\left(t_{i_{I, J}}\right) \bar{x}_{i_{I, J}} \\
& H_{i_{\mathrm{I}, J}}=f_{6}\left(t_{i_{I, J}}\right) \\
& \bar{x}_{i_{I, J}}=\left(\bar{y}_{I, J}+\delta \bar{x}_{I, J}\right) /\left(\delta+m_{H}\left(t_{i_{I, J}}\right)\right) \\
& \left(\bar{i}_{I, J}-\bar{i}_{i_{I, J}}\right) /\left(T_{I, J}-t_{i_{1, J}}\right)=-\gamma C_{H_{I, J}} \\
& t_{I, J+1}=t_{I, J}-\left(h_{G} a X_{e} / G C_{H_{I, J}} N\right)\left(t_{I, J}-t_{i_{\mathrm{I}, J}}\right) \\
& T_{I+1, J}=T_{I, J}-\left(h_{L} a Y_{e} / L C_{P L} M\right)\left(T_{I, J}-t_{i_{\mathrm{I}, J}}\right) \\
& \bar{y}_{I, J+1}=\bar{y}_{I, J}-\left(k_{G} a P X_{e} / G N\right)\left(\bar{y}_{I, J}-\bar{y}_{i_{I, J}}\right) \\
& \bar{x}_{I+1, J}=\bar{x}_{I, J}+\left(k_{L} a \rho_{L M} Y_{e} / L M\right)\left(\bar{x}_{i_{I, J}}-\bar{x}_{I, J}\right) \\
& H_{I, J+1}=H_{I, J}+\left(k_{H} a X_{e} / G N\right)\left(H_{i_{I, J}}-H_{I, J}\right) \\
& C_{H_{I, J}}=C_{a}+C_{b} \bar{y}_{I, J}+C_{v} H_{I, J} \\
& C_{a}=f_{1}\left(t_{I, J}\right), \quad C_{b}=f_{2}\left(t_{I, J}\right), \quad C_{v}=f_{3}\left(t_{I, J}\right) \\
& i_{I, J}=C_{H_{I, J}}\left(t_{I, J}-t_{0}\right)+\left(Q_{0} / \beta\right) \bar{y}_{I, J}+r_{0}(\alpha / \beta) H_{I, J}
\end{aligned}
$$

with the boundary conditions

$$
\left.\begin{array}{c}
t_{I, 1}=t_{i n}, \quad \bar{y}_{I, 1}=y_{i n}, \quad H_{I, 1}=H_{i n} \\
(I=1,2, \cdots, M) \quad \text { at } \quad X=0 \\
T_{1, J}=T_{i n}, \quad \bar{x}_{1, J}=\bar{x}_{i n} \\
(J=1,2, \cdots, N) \quad \text { at } \quad Y=0
\end{array}\right\}
$$

where

$$
\gamma=h_{L} a / h_{G} a, \quad \delta=k_{L} a \rho_{L M} / k_{G} a P
$$

The mean outlet values can be evaluated by

* Equations (15) and (16) can easily be obtained from Eqs. (12) and (13) by assuming Henry's law and that the interfacial concentration of solvent vapor in the gas phase is independent of that of solute in the liquid phase.

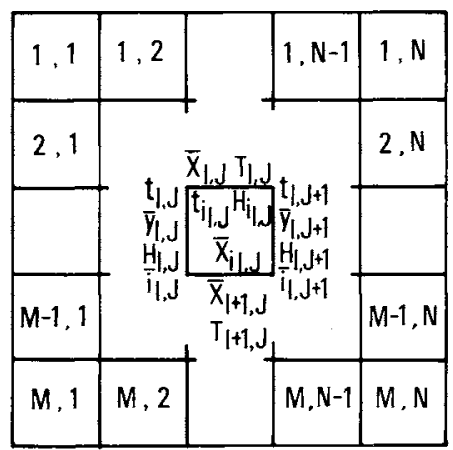

Fig. 2. Representation of the subdivision in a tower.

$$
\begin{aligned}
t_{\mathrm{cal}} & =\sum_{I=1}^{M} t_{I, N+1} / M, \quad \bar{y}_{\mathrm{cal}}=\sum_{I=1}^{M} \bar{y}_{I, N+1} / M \\
H_{\mathrm{cal}} & =\sum_{I=1}^{M} H_{I, N+1} / M, \\
\bar{x}_{\mathrm{cal}} & =\sum_{J=1}^{N} \bar{x}_{M+1, J} / N
\end{aligned}
$$

\section{Calcumation Method for Design Calculation}

The design calculation for the crossflow absorption tower with heat generation can be performed under the given operating conditions as follows:

(A) Estimation of required tower dimensions

(1) Establish operating conditions such as $G, L, t_{i n}$, $\bar{x}_{i n}, \bar{y}_{i n}$ and $H_{i n}$.

(2) Estimate volumetric coefficients, $k_{G} a, k_{H} a, k_{L} a$, $h_{G} a, h_{L} a$, from empirical correlations.

(3) Solve Eqs. (15)-(26) under the assumed values for $X_{e}$ and $Y_{e}$.

(4) Determine the dimensions of the tower, $X_{e}$ and $Y_{e}$, which satisfy the desired outlet conditions.

(B) Estimation of required volumetric coefficients

(1) Establish operating conditions and tower dimensions.

(2) Decide the desired outlet conditions.

(3) Solve Eqs. (15)-(26) under the assumed values for $k_{G} a, k_{H} a, k_{L} a, h_{L} a$ and $h_{G} a$.

(4) Estimate volumetric coefficients which satisfy the desired outlet conditions.

In these cases, unknown variables, $X_{e}, Y_{e}$ or $k_{G} a$, $k_{H} a, k_{\mathrm{L}} a, h_{\mathrm{G}} a, h_{\mathrm{L}} a$, are obtained by a trial-and-error method using Eqs. (15)-(26). Calculations are repeated until the calculated outlet conditions agree with the desired ones within an allowable error. To minimize computation time, the Complex method ${ }^{1)}$ is applied to the present method and the NewtonRaphson method is used to solve the simultaneous equations (15)-(18).

Initial values for unknown variables, which are necessary for calculation, are determined by Eq. (30). 


$$
\left.\begin{array}{l}
\bar{U}_{j}=\ln U_{j, \min }+R A N\left(\ln U_{j, \max }-\ln U_{j, \min }\right) \\
U_{j}=\exp \left(\bar{U}_{j}\right)
\end{array}\right\}
$$

where $U_{j}$ 's correspond to unknown variables to be determined and $R A N$ is a pseudo-random number which is uniformly distributed over the interval $[0,1]$. The criterion function for the minimization is

$$
\begin{array}{r}
F=\operatorname{Max}\left(\frac{\left|t_{\mathrm{set}}-t_{\mathrm{cal}}\right|}{t_{\mathrm{set}}}, \frac{\left|T_{\mathrm{set}}-T_{\mathrm{cal}}\right|}{T_{\mathrm{set}}}, \frac{\left|\bar{x}_{\mathrm{set}}-\bar{x}_{\mathrm{cal}}\right|}{\bar{x}_{\mathrm{set}}},\right. \\
\left.\frac{\left|H_{\mathrm{set}}-H_{\mathrm{cal}}\right|}{H_{\mathrm{set}}}, \frac{\left|\bar{y}_{\mathrm{set}}-\bar{y}_{\mathrm{cal}}\right|}{\bar{y}_{\mathrm{set}}}\right)
\end{array}
$$

In these calculations, the concentration or temperature profile at the gas-liquid interface, the concentrations of solute gas and solvent vapor in the mixed gas and the concentration of solute in the liquid are also estimated. The same charts presented by Inazumi et $a l^{4)}$ are then easily obtained without any troublesome procedure.

\section{Nomenclature}

$$
\begin{aligned}
& \text { a } \quad=\text { effective contact area per unit volume } \quad\left[\mathrm{m}^{2} / \mathrm{m}^{3}\right] \\
& C_{a}, C_{b}, C_{v}=\text { molar heat of inert gas, solute gas and } \\
& \text { solvent vapor respectively }[\mathrm{kJ} /(\mathrm{kmol} \cdot \mathrm{K})] \\
& C_{H} \quad=\text { molar heat of mixed gas defined by Eq. (1) } \\
& {[\mathrm{kJ} /(\mathrm{kmol} \text { of inert gas } \cdot \mathrm{K})]} \\
& C_{P L} \quad=\text { molar heat of solvent } \quad[\mathrm{kJ} /(\mathrm{kmol} \cdot \mathrm{K})] \\
& F \quad=\text { criterion function defined by Eq. (31) } \\
& f_{1}, f_{2}, f_{3}=\text { functions defined by Eq. (11) } \\
& f_{4}, f_{5}, f_{6} \quad=\text { functions defined by Eqs. (12), (13) and (16) } \\
& \text { respectively } \\
& G \quad=\text { mass velocity of inert gas } \quad\left[\mathrm{kmol} /\left(\mathrm{m}^{2} \cdot \mathrm{s}\right)\right] \\
& H \quad=\text { molar humidity } \quad[\mathrm{kmol} / \mathrm{kmol} \text { of inert gas }] \\
& h_{G} \quad=\text { gas-film heat transfer coefficient } \quad\left[\mathrm{W} /\left(\mathrm{m}^{2} \cdot \mathbf{K}\right)\right] \\
& h_{L} \quad=\text { liquid-film heat transfer coefficient } \quad\left[\mathrm{W} /\left(\mathrm{m}^{2} \cdot \mathrm{K}\right)\right] \\
& \bar{i} \quad=\text { modified molar enthalpy of mixed gas defined } \\
& \text { by Eq. (2) [kJ/kmol of inert gas] } \\
& k_{G} \quad=\text { gas-film mass transfer coefficient of solute } \\
& \text { gas } \\
& {\left[\mathrm{kmol} /\left(\mathrm{m}^{2} \cdot \mathrm{s} \cdot \mathrm{Pa}\right)\right]}
\end{aligned}
$$

